\title{
Research on Intelligent Picking-robot System Based on FPGA and Neural Network
}

\author{
Hai-Liu Xiao ${ }^{1, a,{ }^{*}}$, Yan-Ping Wei ${ }^{1, b}$ \\ ${ }^{1}$ Nanchang Institute of Science and Technology, Nanchang,330108,China

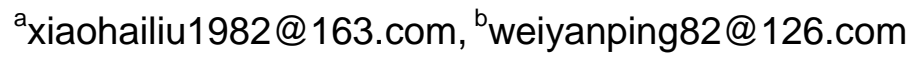

Keywords: FPGA, Neural network, Picking-robot, Intelligent

\begin{abstract}
In order to solve inaccurate picking caused by the nonlinear input and output with the traditional picking measuring robot system, this article will put forward a new robot picking system based on FPGA and neural network. Comparing with the traditional robot system, the new one can enhance the linear relationship between the input and output. By integrating the complex robot control system into FPGA chip, it will not only greatly decrease the cost of development, but also realize the online upgrade and enhance the accuracy of picking by using neural network algorithm. The result shows that the speed of the robot system is very accurate and can greatly improve the efficiency of picking.
\end{abstract}

\section{Introduction}

With the development of the agricultural automatic technology, the picking-robot is used more and more widely and intelligent. Due to the complicated picking environment, the present picking robot more often uses the nonlinear sensor resulting in a nonlinear input and output measuring system which may cause the serious problem, that is the unsatisfactory performance in picking[1]. In order to solve the problem, this article presents the method of simultaneous compensation of the software and hardware. By integrating the whole system into a FPGA chip, this system can realize the joint of the FPGA system and the peripheral interface circuit by software programming. Comparing with the traditional system, the new one can greatly improve the utilization of the computation resource with low cost. Meanwhile, due to short development cycle of the new system, it can realize online upgrade [2].

\section{The general design of the intelligent picking robot}

The largest advantage of the intelligent picking robot is the strong abilities of computing and moving. But its shortcoming is also obvious that is its weakness in awareness. In order to enhance its awareness, wireless network and sensors can be applied. If picking environment is over complicated, real-time monitoring can be adopted to realize the computation and communication task. In this way, its path planning ability can be improved which will achieve high efficiency and more accurate picking [3].

In the figure 1, FPGA is the core of the whole robot. Its main functions are to carry out the data processing and complete the coordination between submodules. There is a HD camera on the forearm of the mechanical arm, which functions as the two eyes of the robot. It can realize the accurate picking positioning of mechanical arm. The movement and picking can be realized with FPGA control. If there are obstacles during its movement, the FPGA system can automatically calculate the distance and control its speed and direction. In this way, it realizes the successful bypass of the obstacles and continues its picking work[4].

\section{The design of its construction and control algorithm}

The control of the selected sensor can be expressed with equation (1):

$y=f\left(x, t_{1}, t_{2}, \cdots, t_{k}\right)$ 
In equation (1), $X$ means the parameter to be measured, and t1,t2, $\cdots$ tk refer to the non target parameter. All the input variables of the sensor are nonlinear, which can approach to linear function with controlling algorithm, therefore, the decreased error can be realized by adjusting the related parameters[5].

In the real application, the distance between the picking robot and the object is measured with triangulation method, through the refraction of the infrared sensors to realize the real time distance measurement of the obstacles. Position of PSD (Position Sensitive detector) is shown in figure 2.
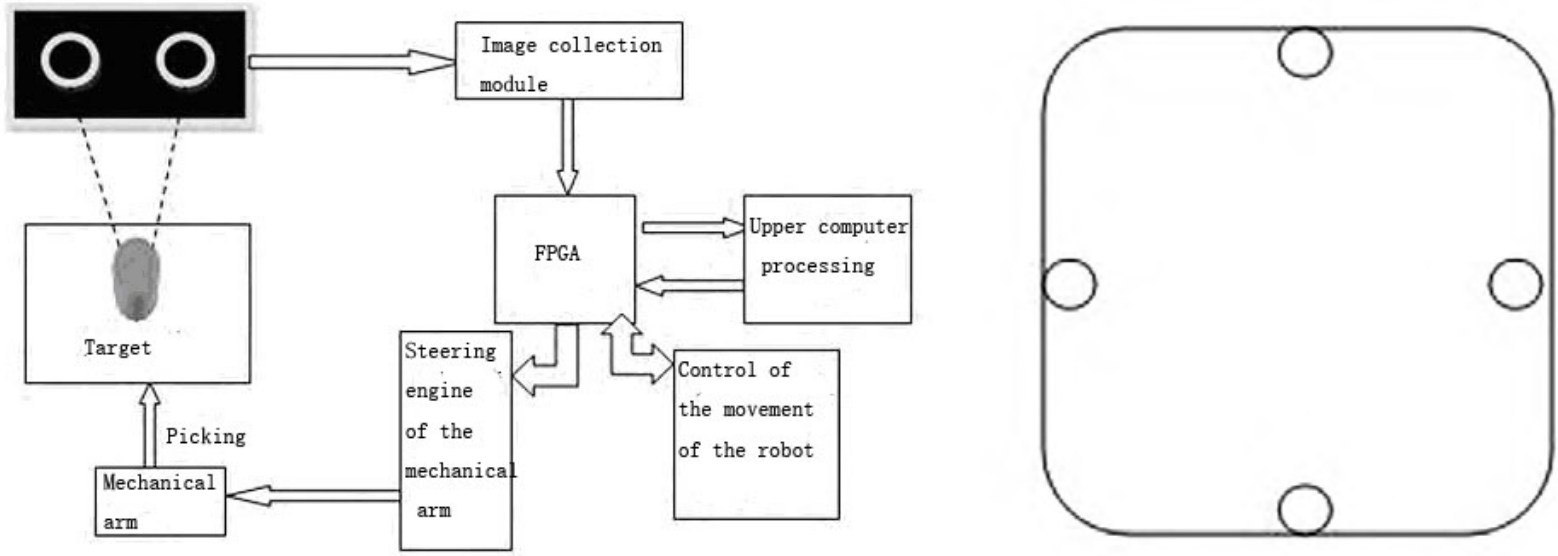

Fig.1 General design of the intelligent picking robot Fig.2 Schematic diagram of position of PSD The control principle of the steering engine with the FPGA system is shown in figure 3.

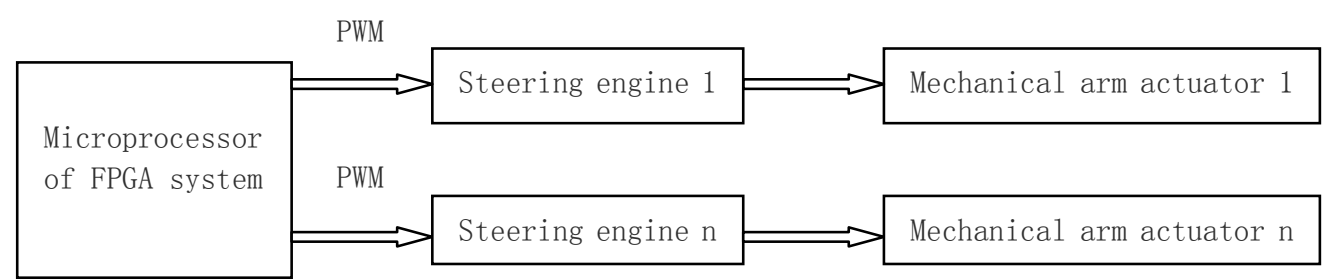

Fig.3 The executive principle of the FPGA steering engine

FPGA gives the command to control the rotation of the motor, then it sends the signal to the steering wheel after the change of the speed. In the design of the hardware, the output bearing and the feedback potentiometer of the motor are connected together, so when the steering works, it will drive the feedback potentiometer to work. The speed and direction of the whole motor are controlled by the feedback signals, at last it realizes its stop [6]. In order to improve its accuracy, it must enhance the accuracy of the robot picking, which can be acquired by appropriate algorithm. On the practical needs, this article selects the BP neurotic network algorithm. Based on the concrete operating status, PID regulator can realize the optimizing process of its movement accuracy, which can be displayed in figure 4.This system can greatly improve the picking accuracy of the robot.

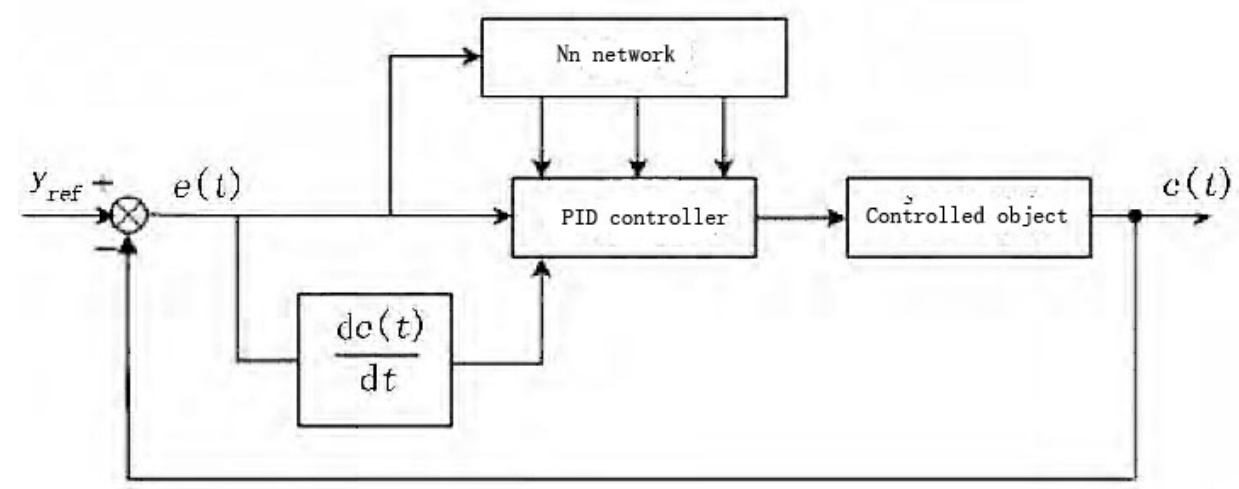

Fig.4 The construction of the neurotic network PID controller based on BP 
In order to acquire the expected goal, it must carry out the discretization to the controlled objective. Meanwhile, the system should be optimized to the system with PID regulator. It can be expressed with the following function.

$$
\begin{aligned}
u(k)= & u(k-1)+[\operatorname{error}(k)-\operatorname{error}(k-1)]+k_{i} \operatorname{error}(k)+k_{d}[\operatorname{error}(\mathrm{k})- \\
& 2 \operatorname{error}(k-1)+\operatorname{error}(k-2)]
\end{aligned}
$$

In this equation, $\mathrm{kp} 、 \mathrm{ki}$ 、 $\mathrm{kd}$ refer to the integration constant, $\mathrm{u}(\mathrm{k})$ the controlled function, and error(k) the controlled error.

Therefore, the input layer of the neurotic network can be expressed with the following quation.

$$
o_{j}^{(1)}=u(j) \quad(j=1,2, \cdots M)
$$

In the above equation, $\mathrm{M}$ is the complex rate of the control system. The input and output of the hidden layer are as the following.

$$
\begin{aligned}
& \operatorname{net}_{i}^{(2)}(k)=\sum_{j=0}^{M} w_{i j}^{(2)} O_{J}^{(1)} \\
& O_{i}^{(2)}(k)=f\left[\operatorname{net}_{i}^{(2)}(k)\right] \quad(i=1, \cdots, Q)
\end{aligned}
$$

In equation (4), wij(2) is the waiting coefficient.If the Sigmoid function is fetched from the neurotic active function, the equation (5) can be acquired:

$$
f(x)=\tanh (x)=\frac{e^{x}-e^{-x}}{e^{x}+e^{-x}}
$$

The input and output of the output layer can be acquired from Equation (6).

$$
\begin{aligned}
& n e t_{l}^{(3)}(k)=\sum_{i=0}^{Q} w_{l i}^{(3)} O_{i}^{(2)}(k) \\
& O_{i}^{(3)}(k)=g\left(\text { net }_{l}^{(3)}(k)\right) \quad(l=1,2,3) \\
& O_{i}^{(3)}(k)=k_{p} \\
& O_{i}^{(3)}(k)=k_{i} \\
& O_{i}^{(3)}(k)=k_{d}
\end{aligned}
$$

And kp、ki 、 kd can also be adjusted

The Sigmoid function of the output layer can be expressed with function (7).

$$
g(x)=\frac{1}{2}[1+\tanh (x)]=\frac{e^{x}}{e^{x}+e^{-x}}
$$

The performance index function can be expressed with equation (8).

$$
E(x)=\frac{1}{2}\left[y_{\text {ref }}(k) \mid-\operatorname{yout}(k)\right]^{2}
$$

In the calculation, the correct method must be used to amend the weight coefficient of the neurotic network. The gradient descent method is adopted in the study, the search and adjust of $\mathrm{E}(\mathrm{k})$ are carried out to the negative direction of the subcoefficient. If a faster rate of convergence to be acquired, a minimum inertia term should be added. Therefore, there is the following equation.

$$
\Delta w_{l i}^{(3)}(k)=-\eta \frac{\partial E(k)}{\partial w_{l i}^{(3)}}+\alpha \Delta w_{l i}^{(3)}(k-1)
$$

In (9), $\eta \quad$ is learning speed, and a is the inertia coefficient.

$$
\frac{\partial E(k)}{\partial w_{l i}^{(3)}}=\frac{\partial E(k)}{\partial y(k)} \cdot \frac{\partial y(k)}{\partial u(k)} \cdot \frac{\partial u(k)}{\partial O_{l}^{(3)}(k)} \cdot \frac{\partial O_{l}^{(3)}(k)}{\partial n e_{l}^{(3)}(k)} \cdot \frac{\partial n e t_{l}^{(3)}(k)}{\partial w_{l i}^{(3)}(k)} \cdot \frac{\partial n e t_{l}^{(3)}(k)}{\partial w_{l i}^{(3)}(k)}=O_{i}^{(2)}(k)
$$


In (10), $\frac{\partial y(k)}{\partial u(k)}$ is not known, during calculation, we should use $\operatorname{sgn}\left(\frac{\partial y(k)}{\partial u(k)}\right)$ to replace it, and we can acquire the calculation of the accuracy with the adjusting of parameter of $\eta$.

$$
\begin{aligned}
& \frac{\partial u(k)}{\partial O_{1}^{(3)}(k)}=\operatorname{error}(k)-\operatorname{error}(k-1) \\
& \frac{\partial u(k)}{\partial O_{2}^{(3)}(k)}=\operatorname{error}(k) \\
& \frac{\partial u(k)}{\partial O_{3}^{(3)}(k)}=\operatorname{error}(k)-2 \operatorname{error}(k-1)+\operatorname{error}(k-2)
\end{aligned}
$$

With functions of (9), (10) and (11), the learning algorithm of the neurotic output layer could be concluded which can be expressed as

$$
\begin{aligned}
& \Delta w_{l i}^{(3)}(k)=-\eta \delta_{l}^{(3)} O_{i}^{(2)}(k)+\alpha \Delta w_{l i}^{(3)}(k-1) \\
& \delta_{l}^{(3)}=\operatorname{error}(k) \operatorname{sgn}\left(\frac{\partial y(k)}{\partial u(k)}\right) \frac{\partial u(k)}{\partial O_{l}^{(3)}(k)} g\left[n e t_{i}^{(3)}(k)\right] \quad(l=1,2,3)
\end{aligned}
$$

In the same way, the weighting coefficient algorithm of the hidden layer is concluded as equation (13).

$$
\begin{aligned}
& \Delta w_{l i}^{(2)}(k)=-\eta \delta_{i}^{(2)} O_{j}^{(1)}(k)+\alpha \Delta w_{i j}^{(2)}(k-1) \\
& \delta_{l}^{(2)}=f^{\prime}\left[n t_{i}^{(2)}(k)\right] \sum_{i=1}^{3} \delta_{l}^{(3)} w_{l i}^{(3)}(k) \quad(i=1,2, \cdots Q) \\
& g^{\prime}(\cdot)=g(x)[1-g(x)], f^{\prime}(\cdot)=\left[1-f^{2}(x)\right] / 2
\end{aligned}
$$

In simulation with software, the controlled object use discretization. The robot system acquires signals of accurate distance through the real time feedback of the obstacles displacement. The system can automatically adjust itself to acquire more precise data with the acquired signals.

\section{Results}

In order to test the views of the article, the test are carried out on two aspects to testify the picking robots performance.

First of all, the control of the velocity is tested. When tests begin, different obstacles are designed as well as the input and output variables. The purpose is to train the three layers of neurotic network [9]. Meanwhile, the specific values are designed to the input, hidden and output layers of the neurotic network node. These values are 18, 28 and 16 respectively. With the algorithm discussed in the article, there comes the velocity curve with the change of time and figure 5 is the result.

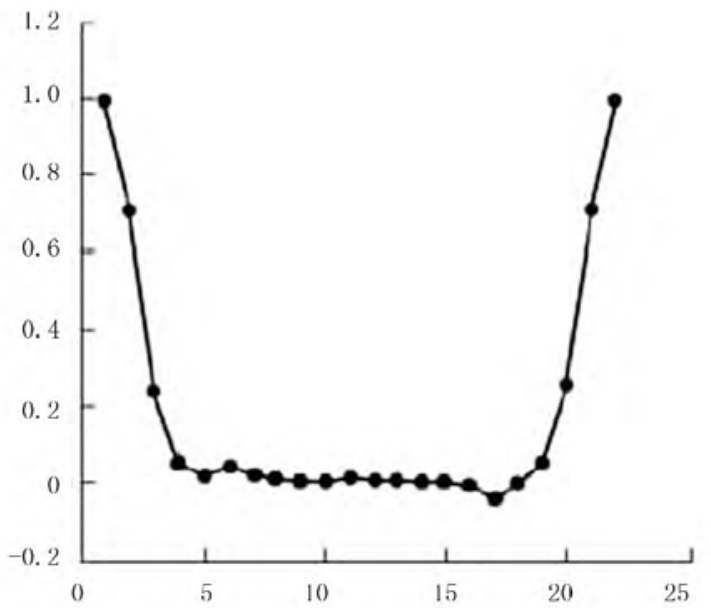

Fig.5 The curve of velocity with the change of time

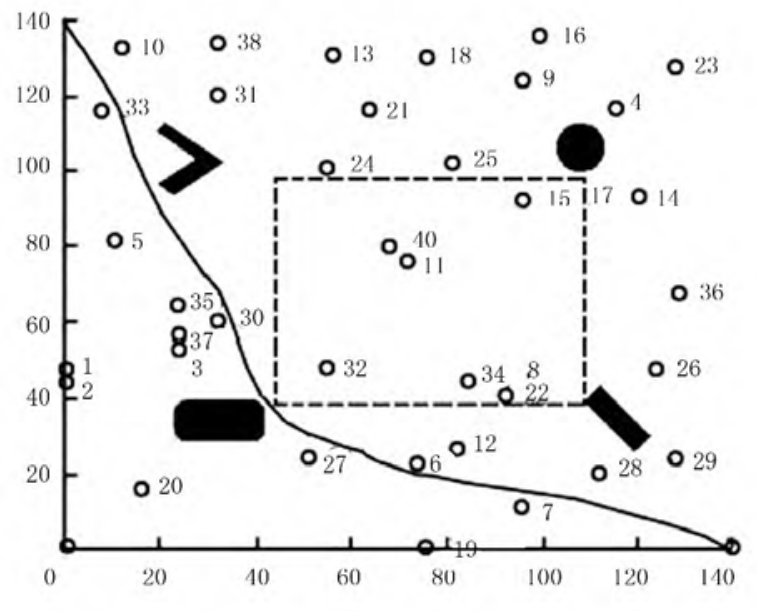

Fig.6 The moving path of the robot 
With the analysis of figure 5, a conclusion can be drawn. If the robot meets an obstacle, it will decrease its speed. When it decreases to some extent, it will adjust its direction automatically and then it will bypass the obstacle. During this process, its control of speed is very accurate and stable as well [10].

Secondly, the test is carried out on its control of the avoidance of the obstacles and movement error. In test, Those 80 collected samples data are put into the neurotic network, at the same time, starting the training. Through the analysis of the result, the moving path is acquired as the following figure 6.

The analysis of figure 6 shows that the robot can successfully bypass obstacles and reach the predesigned place. The article collects the errors when the FPGA system applying the algorithm not the neurotic network. After analysis and calculation, different convergence errors are acquired, the more specific results are displayed in the following two figures( figure 7 and figure 8).

After the analysis of figure 7 and figure 8, we can draw a conclusion that the residual error could be converged more easily with neurotic network algorithm comparing with the general algorithm. This means that the algorithm discussed in the article is correct and reliable.

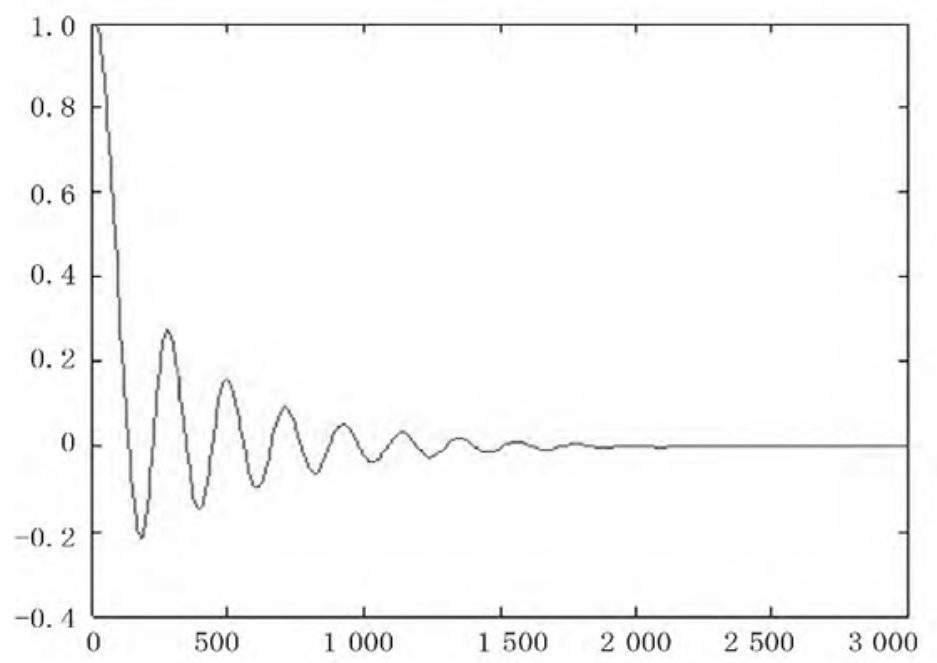

Fig.7 The curve of the residual error with the general algorithm

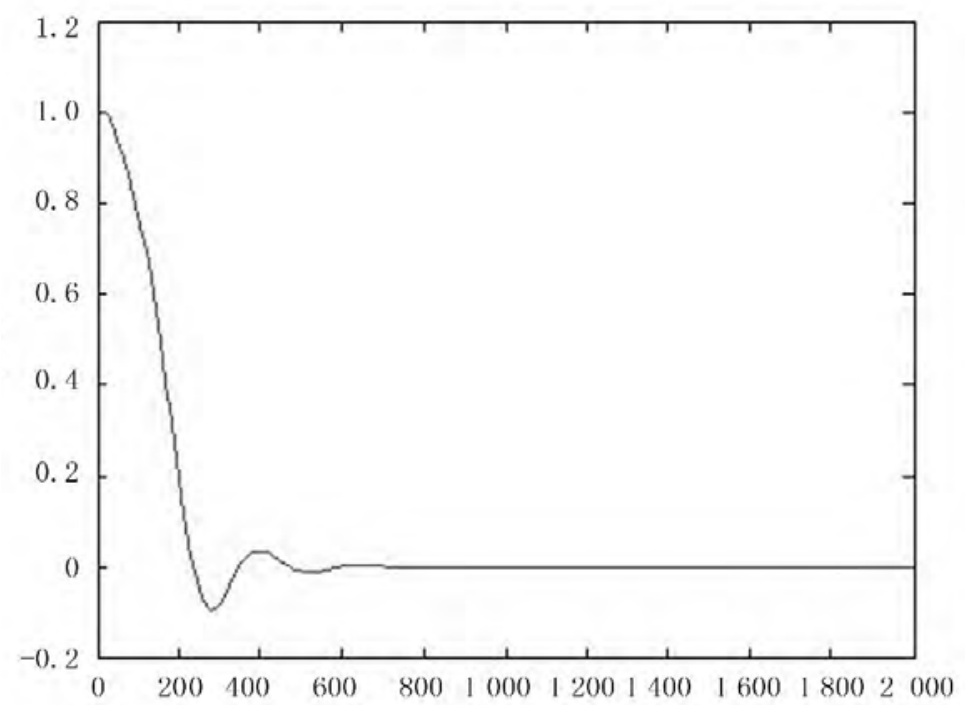

Fig.8 The curve of the residual error with the neurotic network algorithm

\section{Conclusion}

This study has put forward a new picking robot system with the application of FPGA control system and neurotic network PID control algorithm. Comparing with the traditional system, it can better strengthen input and output linear relationship. The integration of the complex control system 
into a FPGA chip, it has greatly decreased the developing cost and can realize the online upgrade. Meanwhile, with the application of neurotic network algorithm can also greatly enhance the picking accuracy.

In order to testify the validity of the new system, this article has carried out the validity test.The result shows that the moving velocity of the robot system is very accurate and it can greatly improve the picking efficiency.

\section{References}

[1]Feng Zhihui, Liu enhai \& Yue Yongjian. The Semiconductor Measuring Distance based on FPGA Delay line interpolation method[J].Journal of Optoelectronics,2011(4):53-59.

[2]Hu Xiangfeng \& Wang Liming. Design of High-speed Data Acquisition System Based on SOPC Technology[J].Journal of Microelectronics and Computer,2009(6):62-65.

[3]Zhao Qingtian, Wei Guangjun \& Yaoyi.Design of the Multi Path Parallel Synchronous digital signal acquisition system Based on SOPC[J].Journal of Ordnance Engineering College,

2011(3):30-65.

[4]Zhang Jin, Wang Boxiong, Cui Yuanyuan,Liu Jiannan \& Zhang Lixin. Flight time The Measurement and Realization of the Flight Time of High Precision Echoes[J].Journal of China Ordnance,2011(8):970-974.

[5]Zhang Meiyu, Huang Han \& Hao Zhifeng.Robot Path Planning Based on Ant Colony Algorithm[J].Computer Engineering and Application, 2005(25):34-37.

[6]Kong Lingjun, Zhang Xinghua \& Chen Jianguo. The Basic Ant Colony Algorithm and Its Improvement[J].Journal of North China University: Natural Science Edition,2004(6):572-574.

[7]Luo Delin \& Wu Shunxiang. Robot Path Planning Based on Potential Field of Ant Colony Algorithm[J],2010(6):1277-1280 .

[8]Xu Sijun \& Cao Qiying.Robot Path Planning Based on Visible Graph[J ],Computer Applications and Software, 2011(3):220-222.

[9]Yin Jianjuan, Wu Chuanyu \& Yang Simon X.Manipulartor Obstacle Avoidance Path Planning of the Tomato Picking Robot[J].Transactions of the Chinese Society for Agricultural Machinery,2012 (12):171-175.

[10]Ji Wei, Cheng Fengyi \& Zhao Dean etc..The Method of Apple picking robot Manipulator Based on Improved Artificial Potential Field Obstacle Avoidance[J].Transactions of the Chinese Society for Agricultural Machinery,2013(11): 253-259. 